\title{
PLANEJAMENTO FAMILIAR E MÉTODOS CONTRACEPTIVOS: PERCEPÇÕES DE PUÉRPERAS FRENTE À GRAVIDEZ NA ADOLESCÊNCIA
}

\section{FAMILY PLANNING AND CONTRACEPTIVE METHODS: PUERPERAL PERCEPTIONS FACING ADOLESCENT PREGNANCY}

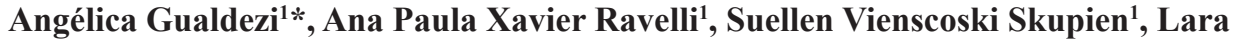 \\ Simone Messias Floriano ${ }^{1}$ \\ ${ }^{1}$ Universidade Estadual de Ponta Grossa, Ponta Grossa, Paraná, Brasil \\ *Autor correspondente: Angélica Gualdezi. Rua Francisco Kmita, 277. Telefone: (42) \\ 991074058. E-mail: angelicagualdezi@hotmail.com
}

\section{RESUMO}

Este artigo trata de pesquisa qualiquantitativa descritiva que objetiva revelar o conhecimento de puérperas adolescentes participantes do projeto Consulta de Enfermagem no Pré-Natal e Pós-Parto sobre planejamento familiar e métodos contraceptivos. As participantes foram 10 (dez) adolescentes vivenciando o Pós-Parto. Foi aplicado questionário estruturado e a análise de conteúdo foi realizada segundo Bardin. Os resultados caracterizaram as adolescentes com idade média de 16,9 anos, a maioria solteiras, que abandonaram os estudos e não trabalhavam. A primeira relação sexual deu-se com média de idade em 14,7 anos, e, quanto ao perfil obstétrico, destacamse as primíparas e parto normal, pré-natal com mais de 6 consultas. O planejamento da gestação foi realizado por $50 \%$ das adolescentes, porém não souberam definir o que é planejamento familiar - as que souberam não envolveram sexualidade segura. $\mathrm{O}$ conhecimento dos métodos contraceptivos chegou a todas as adolescentes, destacandose o anticoncepcional oral e o preservativo masculino. Cabe aos profissionais da saúde, portanto, acolher os adolescentes na atenção primária visando seduzi-los para inserção aos programas de planejamento familiar para sexualidade saudável, minimizando riscos ao surgimento de Doenças Sexualmente Transmissíveis e gravidez não planejada.

Palavras-chave: Enfermagem. Adolescente. Planejamento Familiar.

\begin{abstract}
This paper is about a descriptive qualitative and quantitative research which aimed to reveal the knowledge of teenage mothers participating in the Nursing Consultation project during prenatal and postpartum on family planning and contraception. The participants were 10 (ten) adolescents living postpartum. A structured questionnaire was applied and the content analysis was performed according Bardin. The results characterized the adolescents with an average age of 16.9 years old, most of them single, who were school dropouts and were not working. The first sexual intercourse occurred in an average age of 14.7 years old, and about the obstetric profile we emphasize the primiparous and normal childbirth, prenatal with more than 6 consultations. The planning of pregnancy was carried out by $50 \%$ of the adolescents, however they were not able to define family planning - the ones who knew did not involve safe sexuality. The knowledge of contraceptive methods came to all adolescents, highlighting oral contraceptive and condom. Therefore, it is up to the nursing professionals to welcome adolescents into primary care in order to seduce them into family planning programs to have a healthy sexuality, minimizing risks to the occurrence of Sexually Transmitted Diseases and unplanned pregnancy.

Keywords: Nursing. Adolescent. Family Planning.
\end{abstract}




\section{INTRODUÇÃO}

A adolescência é uma fase rica em possibilidades, mudanças e questionamentos, momento que o indivíduo busca descobrir o mundo que o cerca e se autodescobrir, frente às modificações corpóreas e emocionais. A adolescência é o período dos 10 aos 19 anos, considerando que, a partir dos 10 anos, iniciam-se as transformações físicas, sociais, emocionais e afetivas (BRASIL, 2010).

É nesse período que ocorre a transição da infância para a idade adulta, quando se inicia o desenvolvimento da sexualidade de relevante importância para o crescimento do adolescente em direção à sua identidade adulta, determinando seu caráter, sua autoestima, suas relações afetivas e seu modo de inserção na sociedade (HIGARASHI et al., 2011). Nesse contexto, começam as mudanças sexuais e hormonais, caracterizada pela busca do relacionamento interpessoal, sendo, também, o momento da primeira relação sexual. "Os processos de descobertas do corpo, as mudanças hormonais, a constituição de vínculos, o estabelecimento de sentimentos e desejos na adolescência colaboram para a exacerbação da sexualidade, algo que é íntimo e pessoal" (ARAÚJO et al., 2012, p. 438).

$\mathrm{O}$ adolescente, muitas vezes, tem dificuldade para entender a sua sexualidade, e, na busca desse conhecimento, acaba iniciando sua vida sexual sem orientações, o que pode vir a acarretar em uma gravidez não planejada. Nessa perspectiva, os profissionais de saúde precisam estar preparados para receber tais adolescentes, acolhendo e oferecendo educação em saúde, utilizando uma linguagem apropriada com dinâmicas atrativas. Dessa forma, o planejamento familiar acontecerá com autonomia, pois esses jovens irão apropriar-se de informações, para, assim, optarem conscientemente pela escolha dos métodos contraceptivos gratuitos (BRASIL, 2011).

Muitos profissionais da saúde, porém, encontram-se despreparados para trabalhar com os adolescentes e demonstram preocupação ao desenvolver orientações, pois eles precisam buscar estratégias metodológicas, ou seja, ferramentas e meios didáticos criativos para atrair os adolescentes, procurando valorizá-los, produzindo um cuidado integral de prevenção, promoção e recuperação da saúde (COSTA et al., 2010).
É relevante ressaltar que, na adolescência, há ocorrência de gravidez não planejada, mesmo com a oferta de educação em saúde na atenção primária, bem como educação sexual nas escolas e no contexto familiar. Destaca-se que a gravidez é um período de transformações, transição e amadurecimento, em que o papel de mãe e mulher se alterna, fazendo com que a adolescente precise de apoio familiar e de um companheiro que esteja the dando segurança em relação à nova vida que está por vir (BRAGA et al., 2010). No entanto, na adolescência, nem sempre há o apoio familiar e, muitas vezes, a gestante é abandonada pelo parceiro.

Diante disso, destaca-se aqui o profissional enfermeiro, que precisa estar acessível, ajudando os adolescentes a compreenderem o período vivido, ou seja, a gestação, pois a frequência nas consultas de pré-natal previne possíveis complicações, e, por isso, a relevância em sensibilizar e motivar as gestantes jovens para a busca precoce dos serviços de saúde (VILARINHO; NOGUEIRA; NAGAHAMA, 2012). Dessa forma, os profissionais da atenção primária precisam utilizar estratégias para acolher a gestante adolescente, a fim de criar vínculo, visando seu retorno nas próximas consultas. É importante a inserção ao programa de pré-natal, para que, paulatinamente, ocorra o entendimento sobre as mudanças corporais que acometem a gravidez, bem como orientação quanto ao seu parto e pós-parto, sanando suas dúvidas. Nessa perspectiva, após o parto, a adolescente/puérpera precisará reafirmar seus conhecimentos adquiridos no pré-natal, frente aos cuidados consigo e com o bebê, bem como sua inserção no programa de planejamento familiar.

A hipótese que sustenta esta pesquisa é, portanto, que a promoção da saúde, por meio de ações educativas voltadas aos adolescentes, enfatizando o planejamento familiar e os métodos contraceptivos, podem minimizar o surgimento de gravidez não planejada. Assim sendo, o objetivo deste estudo foi revelar o conhecimento de puérperas adolescentes participantes do projeto Consulta de Enfermagem no Pré-Natal e Pós-Parto (CEPP) sobre o planejamento familiar e os métodos contraceptivos.

\section{METODOLOGIA}

Trata-se de uma pesquisa qualitativa e quantitativa, descritiva, desenvolvida em um Hospital e Maternidade de referência ao atendimento aos partos 
de risco habitual na cidade de Ponta Grossa - Paraná. As participantes foram 10 adolescentes nomeadas como "A" e numeradas de 1 a 10 (A1, A2, A3, A4, A5, A6, A7, A8, A9 e A10).

O critério de inclusão foi a faixa etária dos 15 aos 19 anos e serem participantes do projeto Consulta de Enfermagem no Pré-Natal e Pós-parto (CEPP) vinculado à Universidade Estadual de Ponta Grossa (UEPG), curso de Bacharelado em Enfermagem. Salienta-se que, no projeto CEPP, há atuação de acadêmicas da terceira e quarta série do curso, no qual realizam educação em saúde sobre pós-parto e aleitamento materno.

A coleta dos dados aconteceu por meio de entrevista, com questionário estruturado, utilizado pelo projeto CEPP, bem como questionário semiestruturado com questões norteadoras. As participantes foram entrevistadas de forma individual, em local arejado e privativo, e o questionário semiestruturado foi registrado em gravador digital. A entrevista deve ter um caminho traçado para que se consiga as respostas desejadas com grandes detalhes, assim consegue-se um diálogo enriquecido com a participação de ambas as partes (TRIVIÑOS, 2001).

Os dados advindos da coleta foram analisados por meio da análise de conteúdo segundo Bardin (1977), organizados em três fases: a pré-análise, a exploração do material e o tratamento dos resultados, inferência e interpretação. Para subsidiar os dados advindos do questionário estruturado, foi utilizado somente cálculo de frequência simples.

O projeto foi aprovado pelo Comitê de Ética em Pesquisa da UEPG e contemplou os requisitos éticos em pesquisa envolvendo seres humanos com o Termo de Consentimento Livre e Esclarecido (TCLE). Destaca-se que os responsáveis pelas adolescentes estavam cientes da pesquisa. Os aspectos éticos foram assegurados contemplando a Resolução 466/2012, com parecer do Comitê de Ética e Pesquisa (COEP) 1.055.927, de 8 de maio de 2015, pela UEPG.

\section{RESULTADO E DISCUSSÃO}

Para caracterizar o perfil das participantes do estudo, destacam-se algumas variáveis, como idade: $10 \%$ das puérperas adolescentes tinham 15 anos; $40 \%$ delas, 16 anos; 20\%, 17 anos; 10\% tinham 18 anos; e 20\% delas, 19 anos. A média de idade, assim, foi de 16,9 anos. Estudos que revelam o perfil de gestantes adolescentes possuem uma média semelhante ao encontrado em relação à idade, sendo de 16,7 anos (CAMINHA et al., 2012; GRADIM; FERREIRA; MORAES, 2010).

Dessas adolescentes, apenas $10 \%$ delas relataram ser casadas e $10 \%$ delas convivem com os parceiros - as demais ( $80 \%)$ informaram estar solteiras. A pesquisa de Oliveira, Viera e Fonseca (2011) evidenciou que $58 \%$ das adolescentes moravam com um companheiro e as demais (42\%) disseram estar solteiras.

Já, quanto ao nível de escolaridade, pôde-se desvelar que $10 \%$ das adolescentes completaram o Ensino Médio, 20\% completaram o Ensino Fundamental, 20\% abandonaram o Ensino Médio e 50\% delas abandonaram os estudos no Ensino Fundamental. Salienta-se que o abandono dos estudos frente a uma gestação na adolescência é comum. Oliveira, Viera e Fonseca (2011), em seu estudo que analisou o perfil de gestantes adolescentes no interior do Paraná, evidenciaram que $8 \%$ delas completaram o Ensino Médio, 25\% delas não completaram o Ensino Médio e 33\% abandonaram os estudos no Ensino Fundamental. Por sua vez, outro estudo verificou que $40,90 \%$ das adolescentes não conseguiram completar o Ensino Fundamental e apenas 9,10\% possuíam Ensino Médio completo (GRADIM; FERREIRA; MORAES, 2010).

Em relação à variável Trabalho formal, apenas $20 \%$ delas referiram trabalhar como autônomas e $80 \%$ delas não trabalhavam. Destaca-se outra pesquisa em 2012 que encontrou a ocupação de adolescentes, em que $53 \%$ delas eram donas de casa, $36,5 \%$ eram estudantes e $10,5 \%$ trabalhavam em atividades remuneradas (CAMINHA et al., 2012). Por outro lado, outro estudo apontou que $50 \%$ das adolescentes trabalhavam no lar, $42 \%$ eram estudantes e $8 \%$ disseram ter emprego remunerado como doméstica (OLIVEIRA; VIERA; FONSECA, 2011).

Salienta-se, aqui, outra variável que remete ao perfil das adolescentes estudadas - a primeira relação sexual das participantes. $10 \%$ delas iniciaram a vida sexual aos 13 anos; $40 \%$, aos 14 anos; 30\%, aos 15 anos; $10 \%$, aos 16 anos; e 10\%, aos 17 anos, com uma média de 14,7 anos de idade. Comparando tais dados a um estudo de 2011, Hugo et al. Encontraram uma média de 15,7 anos na primeira relação sexual. Atenta-se que a diferença, neste estudo, se dá em 1 ano mais cedo.

Dessa forma, visando caracterizar o perfil obstétrico das adolescentes participantes deste estudo, destaca-se, inicialmente, o número de gestações 
ocorridas nessa fase da vida, no qual denota que $70 \%$ das adolescentes eram primíparas e 30\% multíparas - estas sofreram um aborto na primeira gestação. O estudo de Filamingo, Lisboa e Basso (2012) justifica os dados apresentados, pois evidenciou que $89,7 \%$ das adolescentes eram primíparas e 10,3\% multíparas.

Por outro lado, a realização do pré-natal é importante para o desenvolvimento da gestação. Este estudo desvelou que $90 \%$ das adolescentes fizeram o pré-natal e tiveram mais de 6 consultas, e apenas $10 \%$ não realizaram nenhuma consulta. Uma pesquisa realizada em 2012, sobre a avaliação da qualidade do pré-natal, evidenciou que $13,2 \%$ das adolescentes realizaram de 1 a 3 consultas; 47,7\%, de 4 a 6 consultas; e 39,1\%, de 7 a 10 consultas (VILARINHO; NOGUEIRA; NAGAHAMA, 2012).

Por fim, quanto ao tipo de parto, $80 \%$ tiveram parto normal e $20 \%$ parto cesárea, números semelhantes a outro estudo, o qual aponta que $71,8 \%$ tiveram parto normal e 28,2\%, parto cesárea (FILAMINGO; LISBOA; BASSO, 2012). Outro ponto a ser destacado foi a intercorrência fetal ocorrida com um (10\%) bebê que nasceu prematuro, visto que a mãe não realizou pré-natal. Um estudo que analisou os fatores de risco da gestação na adolescência dá destaque à prematuridade. Os autores encontraram 13,5\% de adolescentes que tiveram seus filhos com menos de 37 semanas de gestação (SANTOS et al., 2014).

\section{PLANEJAMENTO REPRODUTIVO}

O planejamento familiar é importante para a construção de uma família. Ele até mesmo deve ser realizado a fim de evitar uma gravidez indesejada. Contudo, vê-se que ainda é pouco comum, entre adolescentes, essa prática, visto que elas não possuíam o conhecimento do que vem a ser o planejamento reprodutivo. Assim sendo, podemos encontrar gestações não planejadas nessa fase da vida.

Nesse contexto, um estudo apresentou o planejamento entre adolescentes: $25 \%$ disseram ter planejado a gravidez e $75 \%$ apontaram que ela aconteceu de forma inesperada (OLIVEIRA; VIERA; FONSECA, 2011). Entretanto, este estudo revelou que 50\% das adolescentes planejaram a gestação e 50\% não planejaram. No entanto, quando questionadas se planejaram sua gestação e quanto ao conhecimento sobre o que é planejamento familiar, atenta-se que 30\% das adolescentes não souberam conceituar, porém disseram que planejaram a gestação. Para subsidiar tais relatos, um estudo (SILVA et al., 2011) revelou que 41,7\% das participantes apresentaram desconhecimento sobre planejamento familiar, justificando, então, o resultado apresentado neste estudo. O planejamento familiar é o direito de toda pessoa em receber a informação e ter acesso aos recursos que permitam optar livre e conscientemente em ter ou não filhos (SILVA et al., 2011). Os seguintes relatos elucidam o conhecimento sobre planejamento familiar para as adolescentes:

Namora, casa e dai ter filho. (A2).

Ah, ter uma familia, ter filhos,. (A4).

Senta, conversa e vê, será se tá na hora mesmo. (A5).

Quanto mais planeja melhor fica, do que fazer tudo na louca, louca. (A7).

Ah, sei lá, é quando o casal planeja pra ter o filho. (A8).

Nota-se, por meio desses relatos, que as adolescentes enfatizam que planejar advém somente do ato de ter filhos, não envolvendo a sexualidade segura, prevenindo uma gestação indesejada e Doenças Sexualmente Transmissíveis (DSTs). Pesquisa envolvendo gestantes adolescentes destacou que os serviços de saúde não esclarecem e não incluem os adolescentes no planejamento familiar (DANTAS et al., 2013). Cabe aqui elencar, portanto, o papel do enfermeiro junto às adolescentes na inclusão e na orientação em relação ao planejamento familiar, a fim de conscientizá-los da sua importância, ajudando a diminuir o número de gestação não planejada nessa fase da vida.

\section{MÉTODOS CONTRACEPTIVOS: REVELANDO A REALIDADE E CONSTATANDO OS PROBLEMAS}

O conhecimento de adolescentes frente ao uso de métodos contraceptivos é comum. No entanto, a sua utilização não é frequente, o que acarreta, muitas vezes, na gestação não planejada e DSTs. Das adolescentes participantes do estudo, 100\% relataram conhecer os métodos contraceptivos, o que é comum, constatado em outra pesquisa que também encontrou que $100 \%$ das adolescentes tinham conhecimento sobre os métodos contraceptivos (GRADIM; FERREIRA; MORAES, 2010).

Destaca-se, neste estudo, que os contraceptivos mais conhecidos foram o anticoncepcional oral, por 90\% das adolescentes; o preservativo masculino, por $80 \%$. Outros métodos citados foram a injeção, $40 \%$, 
e o dispositivo intrauterino (DIU), $10 \%$. Outro estudo também revela que os adolescentes conhecem mais o preservativo masculino e a pílula (GRADIM; FERREIRA; MORAES, 2010).

As adolescentes possuem informação sobre a necessidade do uso de contraceptivos. Entretanto, não significa que elas têm o conhecimento para se prevenir adequadamente (DIAS; TEIXEIRA, 2010). Diante disso, vê-se que, em algum momento, as adolescentes utilizaram algum método, visto que $20 \%$ não fizeram uso em nenhum momento, porém os relatos a seguir mostram que as adolescentes não dão sequência ao uso do contraceptivo.

Usei camisinha no começo, depois não usei mais. (A1).

O comprimido no começo eu tomava diariamente; dai comecei a esquecer e parei de tomar. (A6).

Tentei tomar pílula mais não deu certo, dá reação. (A7).

A partir dos relatos, cabe destacar que as orientações ainda são falhas. As adolescentes conhecem os métodos contraceptivos, principalmente o preservativo masculino e o anticoncepcional oral, disponibilizados gratuitamente nas Unidades Básicas de Saúde (UBS), porém não os utilizam de forma adequada, realizando sexo não seguro. Assim, os profissionais de saúde, enfocando os enfermeiros, devem trabalhar integralmente com os métodos contraceptivos, mas direcionando a educação em saúde ao casal, pois, muitas vezes, a responsabilidade recai apenas sobre a mulher. Pesquisa aponta que os meninos raramente fazem a opção de utilizar o preservativo, e as meninas sentem, muitas vezes, vergonha de pedir para que eles o usem (MENDES et al., 2011).

O fato de não usar o preservativo, na maioria das vezes, é pelas adolescentes terem um único parceiro, evidenciado neste estudo, visto que $100 \%$ delas tiveram seu filho com seu parceiro fixo. O tempo de permanência com o parceiro antes da gestação foi de $30 \%$ até 1 ano; $40 \%$, de 1 ano a 5 anos; $10 \%$, mais de 5 anos; e $20 \%$ não soube informar há quanto tempo estavam juntos. Entretanto, no momento que surge uma gestação, a adolescente passa por varias transformações, e ter o apoio do parceiro e da família é importantíssimo, minimizando sofrimento e anseios. A ausência do parceiro torna-se um fator negativo, podendo trazer prejuízos físicos, psíquicos e sociais, podendo ser um fator de risco para a mãe e para o bebê (GRADIM; FERREIRA; MORAES, 2010).
Um estudo aponta que a presença do companheiro na gestação dá segurança e estabilidade para a adolescente (BRAGA et al., 2010). Das adolescentes entrevistadas, apenas duas não permaneceram com os parceiros. Destaca-se, aqui, o seguinte relato:

[...] agora não tô com ele. Tava a 4 anos, a gente tinha terminado e descobri com 5 meses que tava grávida; dai eu tinha brigado com ele, agora eu vou atrás dele. (A1).

É importante que a equipe de saúde, aqui destacando o enfermeiro, se evolva com os adolescentes, buscando trazê-los para a unidade de saúde, a fim de mostrar a importância da utilização dos métodos contraceptivos e a responsabilidade do casal. Necessita-se, também, trazer as gestantes e puérperas adolescentes, procurando dar um apoio e sanar as dúvidas frente ao período vivenciado.

\section{CONCLUSÃO}

A caracterização das puérperas deu-se por meio da idade da gestação, a qual se dá cada vez mais cedo. A média de idade foi de 16,9 anos, a maioria das mães eram solteiras e abandonaram os estudos e não trabalhavam. Destaca-se a primeira relação sexual das adolescentes a qual se deu com média de 14,7 anos. O perfil obstétrico levantado revelou que as adolescentes eram na maioria primíparas e tiveram seus filhos por parto normal, realizaram o pré-natal com mais de 6 consultas.

Em relação ao planejamento familiar, vê-se que metade das adolescentes planejou a gestação, no entanto não sabem definir planejamento familiar. Aquelas que o conceituaram não envolveram a sexualidade segura. Salienta-se que as adolescentes tiveram seus filhos com parceiro fixo e a maioria permaneceu com ele. Quanto aos métodos contraceptivos, todas tinham conhecimento, destacando o anticoncepcional oral e o preservativo masculino como os mais conhecidos. A maioria delas, em algum momento, fez uso de algum contraceptivo.

Diante disso, nota-se que as adolescentes necessitam ser inseridas no planejamento familiar, por isso cabe ressaltar o papel do enfermeiro junto aos adolescentes na busca de inseri-los ao planejamento familiar, podendo então orientá-los, ajudando-os a ter uma sexualidade saudável e auxiliando na prevenção da gestação e das DSTs. Da mesma forma, o enfermeiro 
deve trabalhar junto às gestantes e puérperas procurando tirar as dúvidas que surgem.

\section{REFERÊNCIAS}

ARAÚJO, A. C. et al. Implicações da sexualidade e reprodução no adolescer saudável. Revista Rene, Fortaleza, v. 2, n. 13, p. 437-444, 2012.

BARDIN, L. Análise de conteúdo. São Paulo: Martins Fontes, 1977.

BRAGA, L. P. et al. Riscos psicossociais e repetição de gravidez na adolescência. Boletim de Psicologia, v. 70, n. 133, p. 205-215, 2010.

BRASIL. Ministério da Saúde. Comunicação e Educação em Saúde. Caderneta de saúde do adolescente. 2. ed. Brasília: Ministério da Saúde, 2010.

BRASIL. Portal Brasil. Saúde. Planejamento Familiar. 6 set. 2011. Disponível em: <http://www.brasil.gov.br/ saude/2011/09/planejamento-familiar $>$. Acesso em: 10 nov. 2014.

CAMINHA, N. O. et al. O perfil das puérperas adolescentes atendidas em uma maternidade de referência de FortalezaCeará. Escola Anna Nery, Rio de Janeiro, v. 3, n. 16, p. 486-492, jul./set. 2012.

COSTA, R. F. et al. Cuidado de enfermagem ao adolescente: análise da produção científica de 2001 a 2007. Ciência Cuidado e Saúde, Maringá, v. 3, n. 9, p. 585-592, jul./set. 2010.

DANTAS, L. A. et al. O desabrochar das flores: opiniões de adolescentes grávidas sobre planejamento familiar. Cogitare Enfermagem, Curitiba, v. 3, n. 18, p. 502-508, jul./set. 2013.

DIAS, A. C. G.; TEIXEIRA, M. A. P. Gravidez na adolescência: um olhar sobre um fenômeno complexo. Paideia, Ribeirão Preto, v. 20, n. 45. p. 123-131, jan./abr. 2010.

FILAMINGO, B. O.; LISBOA, B. C. F.; BASSO, N. A. S. A prática do aleitamento materno entre mães adolescentes na cidade de Dois Córregos, Estado de São Paulo. Scientia Medica, Porto Alegre, v. 2, n. 22, p. 81-85, 2012.

GRADIM, C. V. C.; FERREIRA, M. B. L.; MORAES, M. J. O perfil das grávidas adolescentes em uma unidade de saúde da família de Minas Gerais. Revista de APS, Juiz de Fora, v. 1, n. 13, p. 55-61, jan./mar. 2010.

HIGARASHI, I. H. et al. Atuação do enfermeiro junto aos adolescentes: identificando dificuldades e perspectivas de transformação. Revista Enfermagem, Rio de Janeiro, v. 3, n. 19, p. 375-380, jul./set. 2011.
HUGO, T. D. O. et al. Fatores associados à idade da primeira relação sexual em jovens: estudo de base populacional. Caderno Saúde Pública, Rio de Janeiro, v. 11, n. 27, p. 2207-2214, nov. 2011.

MENDES, S. S. et al. Saberes e atitudes dos adolescentes frente à contracepção. Revista Paulista de Pediatria, v. 3, n. 29, p. 385-91, 2011.

OLIVEIRA, B. R. G.; VIERA, C. S.; FONSECA, J. F. N. A. Perfil de adolescentes gestantes de um município do interior do Paraná. Revista Rene, Fortaleza, v. 2, n. 12, p. 238-246, abr./jun. 2011.

SANTOS, N. L. A. C. et al. Gravidez na adolescência: análise de fatores de risco para baixo peso, prematuridade e cesariana. Ciência \& Saúde Coletiva, Rio de Janeiro, v. 3, n. 19, p. 719-726, 2014.

SILVA, R. M. et al. Planejamento familiar: significado para mulheres em idade reprodutiva. Ciência \& Saúde Coletiva, Rio de Janeiro, v. 5, n. 16, p. 2415-2424, 2011.

TRIVIÑOS, A. N. S. Abordagem metodológica do estudo. In: TRIVIÑOS, A. N. S. Bases teórico-metodológicas da pesquisa qualitativa em ciências sociais: ideias gerais para a elaboração de um projeto de pesquisa. v. 4. Porto Alegre: Cadernos de Pesquisa Ritter dos Reis, 2001. p. 85-90.

VILARINHO, L. M.; NOGUEIRA, L. T.; NAGAHAMA, E. E. I. Avaliação da qualidade da atenção à saúde de adolescentes no pré-natal e puerpério. Escola Anna Nery, Rio de Janeiro, v. 2, n. 16, p. 312-319, abr./jun. 2012. 